\title{
ALMOST PERIODIC POTENTIALS IN HIGHER DIMENSIONS
}

\author{
VASSILIS G. PAPANICOLAOU
}

\begin{abstract}
This work was motivated by the paper of R. Johnson and J. Moser (see $[\mathrm{J}-\mathrm{M}]$ in the references) on the one-dimensional almost periodic potentials. Here we study the operator $L=-\Delta / 2-q$, where $q$ is an almost periodic function in $R^{d}$. It is shown that some of the results of [J-M] extend to the multidimensional case (our approach includes the one-dimensional case as well).

We start with the kernel $k(t, x, y)$ of the semigroup $e^{-t L}$. For fixed $t>0$ and $u \in R^{d}$, it is known (we review the proof) that $k(t, x, x+u)$ is almost periodic in $x$ with frequency module not bigger than the one of $q$. We show that $k(t, x, y)$ is, also, uniformly continuous on $[a, b] \times R^{d} \times R^{d}$. These results imply that, if we set $y=x+u$ in the kernel $G^{m}(x, y ; z)$ of $(L-z)^{-m}$ it becomes almost periodic in $x$ (for the case $u=0$ we must assume that $m>d / 2)$, which is a generalization of an old one-dimensional result of Scharf (see [S.G]). After this, we are able to define $w_{m}(z)=M_{x}\left[G^{m}(x, x ; z)\right]$, and, by integrating this $m$ times, an analog of the complex rotation number $w(z)$ of [J-M]. We also show that, if $e(x, y ; \lambda)$ is the kernel of the projection operator $E_{\lambda}$ associated to $L$, then the mean value $\alpha(\lambda)=M_{x}[e(x, x ; \lambda)]$ exists. In one dimension, this (times $\pi$ ) is the rotation number. In higher dimensions ( $d=1$ included), we show that $d \alpha(\lambda)$ is the density of states measure of [A-S] and it is related to $w_{m}(z)$ in a nice way. Finally, we derive a formula for the functional derivative of $w_{m}(z ; q)$ with respect to $q$, which extends a result of $[\mathrm{J}-\mathrm{M}]$.
\end{abstract}

\section{INTRODUCTION. SCHRÖDINGER OPERATORS}

Let $C_{b}\left(R^{d}\right)$ be the class of bounded continuous functions in $R^{d}, d \geq 1$. This class is complete with respect to the supnorm, which we will always denote by $\|\cdot\|$. For a real-valued $q$ in $C_{b}\left(R^{d}\right)$ we define

$$
L=L(q)=-\Delta / 2-q,
$$

where $\Delta$ is the Laplacian operator. $L$ is a Schrödinger operator with potential $-q$. It is well known (see [S.B, Theorem B.12.1], etc.) that $L$, as an operator of $L^{2}\left(R^{d}\right)$, is essentially selfadjoint with a unique selfadjoint extension (with zero boundary conditions at infinity) and $L^{2}$-spectrum $\sigma(L)$ bounded below. In fact, if we set $\lambda_{0}=\inf \sigma(L)$, then $\lambda_{0} \geq-\|q\|$. Furthermore, for each $t>0$, $e^{-t L}$ is a well-defined semigroup. It turns out that this semigroup possesses a kernel $k(t, x, y)$ which is nonnegative, continuous in $(t, x, y)$ if $t>0$,

Received by the editors November 20, 1989.

1980 Mathematics Subject Classification (1985 Revision). Primary 35J10.

Key words and phrases. Almost periodic functions of several variables, Schrödinger semigroup, resolvent, (complex) rotation number, (integrated) density of states. 
symmetric in $(x, y)$ and bounded, provided that $t$ stays in compact subsets of $(0, \infty)$. This kernel is, of course, the fundamental solution (at least in the weak sense) of the parabolic problem that corresponds to $L$, namely

$$
\begin{gathered}
\frac{\partial}{\partial t} k(t, x, y)=\frac{1}{2} \Delta_{x} k(t, x, y)+q(x) k(t, x, y), \\
(t, x, y) \in(0, \infty) \times R^{d} \times R^{d} ; \\
k(0, x, y)=\delta(x-y), \quad(x, y) \in R^{d} \times R^{d} ; \\
\lim _{|x| \rightarrow \infty} k(t, x, y)=0, \quad(t, y) \in(0, \infty) \times R^{d},
\end{gathered}
$$

where $|x|=\sqrt{x_{1}^{2}+x_{2}^{2}+\cdots+x_{d}^{2}}$ and the initial condition (i.e. the second equation above) means that

$$
\lim _{t \downarrow 0} \int_{R^{d}} k(t, x, y) f(y) d y=f(x)
$$

for every $f$ in $C_{b}\left(R^{d}\right)$.

We briefly review the construction of $k(t, x, y)$. First we set

$$
k_{0}(t, x, y)=p_{d}(t, x, y)=\frac{1}{(2 \pi t)^{d / 2}} \exp \left(-\frac{|x-y|^{2}}{2 t}\right)
$$

and, for $n=1,2, \ldots$,

$$
k_{n}(t, x, y)=\int_{0}^{t} \int_{R^{d}} p_{d}(s, x, z) q(z) k_{n-1}(t-s, z, y) d z d s .
$$

Then

$$
k(t, x, y)=\sum_{n=0}^{\infty} k_{n}(t, x, y)
$$

and the convergence of the series is absolute and uniform in $(t, x, y)$, if $t \in$ $[a, b]$, because of $(1.5)$ below. Now, let $B=\left(B_{t}, \mathscr{F}_{t}, P^{x}\right)$ be a (standard) Brownian motion process in $R^{d}$ starting at $x$. The transition density of $B_{t}=$ $\left(B_{t}^{1}, \ldots, B_{t}^{d}\right)$ is the function $p_{d}(t, x, y)$ of $(1.3 \mathrm{a})$. By the Markov property of $B$ and straightforward (rather tedious though) induction on $n$ we get that, if $f \in L^{1}\left(R^{d}\right)$, then

$$
\int_{R^{d}} k_{n}(t, x, y) f(y) d y=\frac{1}{n !} E^{x}\left\{\left[\int_{0}^{t} q\left(B_{s}\right) d s\right]^{n} f\left(B_{t}\right)\right\} .
$$

We can rewrite this formula in an equivalent but more revealing form, with the help of the Brownian bridge (i.e. Brownian motion conditioned to hit a certain point at a given time $t>0$ )

$$
k_{n}(t, x, y)=\frac{1}{n !} E^{x}\left\{\left[\int_{0}^{1} q\left(B_{s}\right) d s\right]^{n} \mid B_{t}=y\right\} p_{d}(t, x, y)
$$

and, therefore,

$$
\left|k_{n}(t, x, y)\right| \leq \frac{1}{n !}(\|q\| t)^{n} p_{d}(t, x, y)
$$


Formulas (1.3c) and (1.4) imply immediately that

$$
k(t, x, y)=E^{x}\left\{e_{q}(t) \mid B_{t}=y\right\} p_{d}(t, x, y),
$$

where $e_{q}(t)$ is the Feynman-Kac functional of $B$ that corresponds to $q$ defined by

$$
e_{q}(t)=\exp \left[\int_{0}^{t} q\left(B_{s}\right) d s\right] .
$$

Now, (1.6) helps us to get a nice estimate for $k(t, x, y)$, namely

$$
e^{-\|q\| t} p_{d}(t, x, y) \leq k(t, x, y) \leq e^{\|q\| t} p_{d}(t, x, y) .
$$

From this construction, the properties of $k(t, x, y)$ mentioned above follow easily. For example, (1.7) implies that $k(t, x, y)$ satisfies the boundary condition at infinity in (1.2) and, furthermore, that, if $1 \leq p \leq q \leq \infty$, then $e^{-t L}$ is in $\mathscr{L}\left(L^{p}, L^{q}\right)$ and in $\mathscr{L}\left(L^{p}, C_{b}\right)$, i.e. the classes of bounded linear operators from $L^{p}\left(R^{d}\right)$ to $L^{q}\left(R^{d}\right)$ and from $L^{p}\left(R^{d}\right)$ to $C_{b}\left(R^{d}\right)$ respectively. Finally, since $k(t, x, y)$ is the kernel of a semigroup, it satisfies the ChapmanKolmogorov equation

$$
k(t+s, x, y)=\int_{R^{d}} k(t, x, z) k(s, z, y) d z .
$$

Now, let $f$ be a Borel function on $\sigma(L)$. The spectral theorem says that

$$
f(L)=\int_{\lambda_{0}}^{\infty} f(\lambda) d E_{\lambda}
$$

in particular

$$
e^{-t L}=\int_{\lambda_{0}}^{\infty} e^{-t \lambda} d E_{\lambda}=t \int_{\lambda_{0}}^{\infty} e^{-t \lambda} E_{\lambda} d \lambda
$$

A very nice result in [S.B, Theorem B.7.1] tells us that, if for some constant $C$

$$
|f(\lambda)| \leq C(1+|\lambda|)^{-\alpha}, \quad \text { where } \alpha>d / 2, \lambda \in \sigma(L),
$$

then $f(L)$ is an integral operator with a jointly continuous and uniformly bounded (integral) kernel. In particular, the projection operator $E_{\lambda}$ has such a kernel which we denote by $e(x, y ; \lambda)$. As a function of $\lambda, e(x, y ; \lambda)$ is constant outside $\sigma(L)$ and vanishes if $\lambda<\lambda_{0}$. Furthermore (see [S.B]), $e(x, x ; \lambda) \geq 0$, and is nondecreasing in $\lambda$. Then, by (1.9) we have (see the appendix for more justifications)

$$
k(t, x, x)=t \int_{\lambda_{0}}^{\infty} e^{-\lambda t} e(x, x ; \lambda) d \lambda=\int_{\lambda_{0}}^{\infty} e^{-\lambda t} d_{\lambda} e(x, x ; \lambda) .
$$

This formula plays a dominant role in the present work. If $\lambda \geq 0,(1.11)$ implies (since $e(x, x ; \lambda)=0$ for $\left.\lambda<\lambda_{0}\right)$

$$
k\left(\lambda^{-1}, x, x\right) \geq \int_{0}^{\lambda} e^{-\mu / \lambda} d_{\mu} e(x, x ; \mu) \geq e^{-1}[e(x, x ; \lambda)-e(x, x ; 0)] .
$$

Thus, by (1.7) and the boundedness of $e(x, y ; 0)$, there are constants $C_{1}$ and $C_{2}$ such that

$$
e(x, x ; \lambda) \leq C_{1} \lambda^{d / 2}+C_{2},
$$


for all $x \in R^{d}$ and $\lambda \geq 0$. The monotonicity in $\lambda$ gives then that, if $\lambda>0$, $\left(1.12^{\prime}\right)$

$$
e(x, x ; \lambda) \leq C_{2} \text {. }
$$

Example. If $q \equiv 0$ (hence $\lambda_{0}=0$ ), the tables for Laplace transforms give us, for $\lambda \geq 0$, (the index indicates the dimension)

$$
\begin{gathered}
e_{1}(x, y ; \lambda)=\frac{\sin (\sqrt{2 \lambda}|x-y|)}{\pi|x-y|}, \\
e_{3}(x, y ; \lambda)=\frac{1}{2 \pi^{2}|x-y|^{3}}[\sin (\sqrt{2 \lambda}|x-y|)-\sqrt{2 \lambda}|x-y| \cos (\sqrt{2 \lambda}|x-y|)]
\end{gathered}
$$

and by elementary properties of the Laplace transform

$$
e_{d+4}(x, y ; \lambda)=\frac{1}{2 \pi^{2}|x-y|^{2}}\left[\pi(d+2) e_{d+2}(x, y ; \lambda)-\lambda e_{d}(x, y ; \lambda)\right]
$$

For even $d, e_{d}(x, y ; \lambda)$ is not an elementary function, but can be expressed in terms of Bessel functions.

Finally, let $D_{N}, N=1,2, \ldots$, be a sequence of bounded domains (open and connected sets) in $R^{d}$ with reasonable (say Lipschitz) boundaries, such that

$$
D_{N+1} \supset D_{N} \text { and } \bigcup_{N} D_{N}=R^{d} \text {. }
$$

For each $N$, we consider the operator $L^{N}=L$ acting on $L^{2}\left(D_{N}\right)$ with zero boundary conditions on $\partial D_{N}$. The corresponding semigroup $e^{-t L^{N}}$ has kernel

$$
k^{N}(t, x, y)=E^{x}\left\{e_{q}(t) 1_{\left[\tau_{N}>t\right]} \mid B_{t}=y\right\} p_{d}(t, x, y)=\sum_{j=0}^{\infty} e^{-\lambda_{j} t} \varphi_{j}(x) \overline{\varphi_{j}(y)},
$$

where $\tau_{N}$ is the (first) exit time of $D_{N}$, namely

$$
\tau_{N}=\inf \left\{t>0: B_{t} \in D_{N}^{c}\right\},
$$

and $\lambda_{0}, \lambda_{1}, \ldots$ are the eigenvalues of $L^{N}$ with corresponding eigenfunctions $\varphi_{0}, \varphi_{1}, \ldots$, which are orthonormal in $L^{2}\left(D_{N}\right)$. The probabilistic form of $k^{N}(t, x, y)$ yields

$$
k^{N}(t, x, y) \uparrow k(t, x, y), \quad \text { as } N \rightarrow \infty,
$$

by dominated convergence, since $1_{\left[\tau_{N}>t\right]} \uparrow 1$ as $N \rightarrow \infty$. then

If $e^{N}(x, y ; \lambda)$ is the kernel of the corresponding projection operator $E_{\lambda}^{N}$

$$
e^{N}(x, y ; \lambda)=\sum_{\lambda_{j} \leq \lambda} \varphi_{j}(x) \overline{\varphi_{j}(y)}
$$

and

$$
k^{N}(t, x, y)=\int_{-\infty}^{\infty} e^{-\lambda t} d_{\lambda} e^{N}(x, y ; \lambda)
$$

In particular, $e^{N}(x, x ; \lambda)$ is nonnegative, nondecreasing and satisfies (1.12) and $\left(1.12^{\prime}\right)$ with constants $C_{1}$ and $C_{2}$ not depending on $N$ or $x$. Thus, by a standard selection argument (see [F, Chapter XIII], the extended continuity theorem), for each $x \in R^{d}$,

$$
\lim _{N} e^{N}(x, x ; \lambda)=e(x, x ; \lambda),
$$

at the points $(\lambda)$ of continuity of $e(x, x ; \lambda)$. 
The jumps of $e(x, x ; \lambda)$ can occur only on the pure point spectrum (i.e. the $L^{2}$-eigenvalues) of $L$, which is a countable set.

\section{THE ALMOST PERIODIC CASE}

We start with some definitions.

Definition 2.I. For $f$ in $C_{b}\left(R^{d}\right)$ and $u$ in $R^{d}$, we set $f_{u}(x)=f(x+u)$, the translate of $f$. We call the function $f$ almost periodic, symbolically $f \in$ $A P\left(R^{d}\right)$, if for any sequence of points $u_{n}, n=1,2, \ldots$, in $R^{d}$, the sequence of functions $f_{u_{n}}$ has a subsequence which converges uniformly in $R^{d}$. If $f$ is a uniform limit of periodic functions, it is called limit periodic.

This is the standard way to define almost periodicity in any locally compact abelian group, in particular in $R^{d}$. Obviously, every continuous periodic function is almost periodic.

Definition 2.II. A function $\varphi$ in $C_{b}\left(R^{d}\right)$ is called a trigonometric polynomial if it has the form

$$
\varphi(x)=\sum_{\xi \in J} e^{i \xi \cdot x},
$$

where $\xi$ is in $R^{d}, J$ is a finite set and $\xi \cdot x=\xi_{1} x_{1}+\cdots+\xi_{d} x_{d}$ is the standard "dot product" in $R^{d}$.

It is easy to see that every trigonometric polynomial is in $A P\left(R^{d}\right)$. Furthermore

Theorem 2.1. A function $f$ in $C_{b}\left(R^{d}\right)$ is almost periodic if and only if it is the uniform limit of a sequence of trigonometric polynomials. In particular, uniform limits of almost periodic functions are almost periodic (and, therefore, every limit periodic function is almost periodic).

The proofs of Theorems 2.1, 2.2, 2.3 and 2.4 can be found in [L] or [S.M].

Theorem 2.2. Let $f \in A P\left(R^{d}\right)$ and $C(T)$ be the cube in $R^{d}$ with side $T$, center at the origin and sides parallel to the axes $x_{1}, \ldots, x_{d}$. Then the limit

$$
\lim _{T \rightarrow \infty} \frac{1}{T^{d}} \int_{C(T)} f(x) d x
$$

exists.

Definition 2.III. The number

$$
M[f]=\lim _{T \rightarrow \infty} \frac{1}{T^{d}} \int_{C(T)} f(x) d x
$$

is called the mean value of $f$. The vectors $\xi$ for which

$$
M_{x}\left[f(x) e^{-i \xi x}\right] \neq 0
$$

generate a module which is called the frequency module of $f$ and is denoted by $F M(f)$. The Fourier series $S[f]$ of $f$ is the (formal) series

$$
\sum_{\xi \in R^{d}} M_{x}\left[f(x) e^{-i \xi x}\right] e^{i \xi x} .
$$


Theorem 2.3. Assume that $f$ is in $A P\left(R^{d}\right)$. Then there is only a countable number of $\xi$ 's for which

$$
M_{x}\left[f(x) e^{-i \xi x}\right] \neq 0
$$

and, therefore, $S[f]$ takes the form

$$
\sum_{n=0}^{\infty} \hat{f}_{n} e^{i \xi_{n} x}
$$

where $\hat{f}_{n}=M_{x}\left[f(x) e^{-i \xi_{n} x}\right]$. There are certain summability properties that $S[f]$ has. In particular, it satisfies the Parseval relation

$$
\sum_{n=0}^{\infty}\left|\hat{f}_{n}\right|^{2}=M\left[|f|^{2}\right] .
$$

Finally, if $f_{n} \rightarrow f$ uniformly in $R^{d}$, then $F M(f) \subset \bigcup_{n} F M\left(f_{n}\right)$.

We need one more definition.

Definition 2.IV. Let $f$ be in $C_{b}\left(R^{d}\right)$ and $\varepsilon>0$. We say that $a \in R^{d}$ is an $\varepsilon$-period of $f$ if

$$
|f(x+a)-f(x)|<\varepsilon, \quad \text { for all } x \in R^{d} .
$$

The following theorem is another standard characterization of almost periodic functions.

Theorem 2.4. A function $f$ in $C_{b}\left(R^{d}\right)$ is almost periodic if and only if, for any $\varepsilon>0$ there is an $r=r(\varepsilon)$ such that, every ball in $R^{d}$ of radius $r$ contains an $\varepsilon$-period of $f$. Furthermore, if it happens that, for every $\varepsilon>0$, every $\varepsilon$-period of $f$ is also an E-period of another continuous function $g$, then $g$ is almost periodic and $F M(g) \subset F M(f)$.

The following interesting result first appeared in [A-S]. The proof we give here is almost trivial, thanks to the probabilistic form of $k(t, x, y)$, as given in (1.6).

Theorem 2.5. Let $q$ be in $A P\left(R^{d}\right)$ and $k(t, x, y)$ be the kernel of its corresponding semigroup. Then, for every fixed $t>0$ and $u \in R^{d}, f(x)=$ $k(t, x, x+u)$ is in $A P\left(R^{d}\right)$ and $F M(f) \subset F M(q)$.

Proof. Let $a$ be an $\varepsilon$-period of $q$. Then (1.6) implies

$$
\begin{aligned}
f(x+a)-f(x)= & E^{x+a}\left\{e_{q}(t) \mid B_{t}=x+a+u\right\} p_{d}(t, x+a, x+a+u) \\
& -E^{x}\left\{e_{q}(t) \mid B_{t}=x+u\right\} p_{d}(t, x, x+u)
\end{aligned}
$$

and, since $B$ is spatially homogeneous,

$$
f(x+a)-f(x)=E^{x}\left\{e_{q_{u}}(t)-e_{q}(t) \mid B_{t}=x+u\right\} \frac{1}{(2 \pi t)^{d / 2}} e^{-|u|^{2} / 2 t},
$$

where $q_{u}(x)=q(x+u)$ as in Definition 2.I. Thus

$$
|f(x+a)-f(x)| \leq \frac{1}{(2 \pi t)^{d / 2}} e^{\|q\|}\left(e^{\varepsilon t}-1\right)
$$

and so, Theorem 2.4 finishes the proof. 
Remark. (a) Clearly, the theorem is also true for each $k_{n}(t, x, y)$ of (1.4).

(b) If $q$ is limit periodic, the theorem implies that $f$ is limit periodic too. Likewise, if $q$ is periodic, so is $f$. In the periodic case and because of the uniqueness of Laplace transforms (see [F, Chapter XIII]), formula (1.11) implies that $e(x, x ; \lambda)$ is also periodic in $x$, whereas, in general, if $q$ is almost periodic, $e(x, x ; \lambda)$ may not be so (see $[\mathrm{J}-\mathrm{M}])$.

For the analysis of $\S 3$, we need to establish a deeper fact about $k(t, x, y)$. We start with two interesting key lemmas. The first appears in [A-S] and its proof follows immediately from the probabilistic form of $k(t, x, y)$ as given in (1.6).

Lemma 2.6. Let $q_{n}, n=1,2, \ldots$, be in $C_{b}\left(R^{d}\right)$ and $k^{n}(t, x, y)$ (here $n$ is a superscript $)$ be the kernel of the semigroup $e^{-t L\left(q_{n}\right)}$. Assume that $\sup _{n}\|q\|=$ $Q<\infty$ and that $q_{n}$ converge to $q \in C_{b}\left(R^{d}\right)$ uniformly on compact sets of $R^{d}$ (respectively uniformly in $R^{d}$ ). Then, for any fixed $t>0, k^{n}(t, x, y)$ converges to the kernel $k(t, x, y)$ that corresponds to $q$, uniformly on compact sets of $R^{d} \times R^{d}$ (respectively uniformly in $R^{d} \times R^{d}$ ).

Proof. Assume that $q_{n} \rightarrow q$ uniformly on compact sets and fix a $t>0$. Now, let $K \subset R^{d}$ be compact. Choose a ball $G$ in $R^{d}$, such that $G \supset K$ and, if $\varepsilon>0$ is given,

$$
\sup _{x, y \in K} P^{x}\left\{\tau_{G}<t \mid B_{t}=y\right\}<\varepsilon,
$$

where $\tau_{G}=\inf \left\{t>0: B_{t} \in G^{c}\right\}$ is the (first) exit time of $G$. Then choose $n_{0}$ such that, for all $n \geq n_{0}$,

$$
\sup _{x \in G}\left|q(x)-q_{n}(x)\right|<\varepsilon .
$$

Thus, by (1.6),

$$
\begin{aligned}
\sup _{x, y \in K} & \left|k(t, x, y)-k^{n}(t, x, y)\right| \\
\leq & E^{x}\left\{\left|e_{q}(t)-e_{q_{n}}(t)\right|, \tau_{G} \geq t \mid B_{t}=y\right\} p_{d}(t, x, y) \\
& +E^{x}\left\{\left|e_{q}(t)-e_{q_{n}}(t)\right|, \tau_{G}<t \mid B_{t}=y\right\} p_{d}(t, x, y) \\
< & \frac{1}{(2 \pi t)^{d / 2}}\left[e^{Q}\left(e^{\varepsilon t}-1\right)+2 e^{Q_{\varepsilon}}\right],
\end{aligned}
$$

which proves that $k^{n}(t, x, y) \rightarrow k(t, x, y)$ uniformly on compact sets of $R^{d} \times$ $R^{d}$, since $K$ and $\varepsilon$ where arbitrary.

The case where $q_{n} \rightarrow q$ uniformly in $R^{d}$ is even easier.

Remarks. (a) In fact the above proof shows that the convergence is uniform in $t$ also, if we restrict it in compact sets of $(0, \infty)$. The assumption $Q<\infty$ is, of course, redundant if $q_{n} \rightarrow q$ uniformly in $R^{d}$.

(b) Lemma 2.6 together with (1.7), imply the following remarkable fact. If $q_{n} \rightarrow q$ uniformly on compact sets of $R^{d}$ and $\sup _{n}\|q\|<\infty$, then $e^{-t L\left(q_{n}\right)} \rightarrow$ $e^{-L(q)}$ in the uniform operator topology of $\mathscr{L}\left(L^{p}, L^{q}\right)$ and $\mathscr{L}\left(L^{p}, C_{b}\right)$, where $1 \leq p \leq q \leq \infty$.

Lemma 2.7. Let $B$ be a Brownian motion in $R^{d}$ starting at $x, f \in L^{p}\left(R^{d}\right)$, $1 \leq p \leq \infty, \gamma_{j}=\alpha_{j}+i \beta_{j} \in C^{d}, j=1, \ldots, n$ and $0 \leq s_{1} \leq s_{2} \leq \cdots \leq s_{n} \leq t$. 
Then

$$
E^{x}\left\{e^{\gamma_{1} \cdot B_{s_{1}}+\cdots+\gamma_{n} \cdot B_{s_{n}}} f\left(B_{t}\right)\right\}=\int_{R^{d}} H\left[s_{1}, \ldots, s_{n} ; \gamma_{1}, \ldots, \gamma_{n}\right](t, x, y) f(y) d y,
$$

where $\gamma \cdot B_{s}=(\alpha+i \beta) \cdot B_{s}=\left(\alpha^{1}+i \beta^{1}\right) B_{s}^{1}+\cdots+\left(\alpha^{d}+i \beta^{d}\right) B_{s}^{d}$ and

$$
\begin{aligned}
H\left[s_{1}, \ldots, s_{n} ; \gamma_{1}, \ldots, \gamma_{n}\right](t, x, y) \\
=\frac{1}{(2 \pi t)^{d / 2}} \prod_{k=1}^{d} E^{x}\left\{e^{\gamma_{1}^{k} B_{s_{1}}^{k}+\cdots+\gamma_{n}^{k} B_{s_{n}}^{k}}\right\} \\
\quad \times \exp \left\{-\frac{1}{2 t}\left[\left(\gamma_{1}^{k} s_{1}+\cdots+\gamma_{n}^{k} s_{n}\right)+\left(x^{k}-y^{k}\right)\right]^{2}\right\},
\end{aligned}
$$

with $x=\left(x^{1}, \ldots, x^{d}\right)$, i.e. $k$ is a superscript, and

$$
E^{x}\left\{e^{\gamma_{1}^{k} B_{s_{1}}^{k}+\cdots+\gamma_{n}^{k} \cdot B_{s_{n}}^{k}}\right\}=e^{\left.\gamma_{1}^{k}+\cdots+\gamma_{n}^{k}\right) x^{k}} \prod_{j=1}^{n} e^{(1 / 2) s_{j} \gamma_{j}^{k}\left(\gamma_{j}^{k}+2 \gamma_{j+1}^{k}+\cdots+2 \gamma_{n}^{k}\right)}
$$

Proof. First we notice that it is enough to prove the formulas only for $f$ 's of the form $f(x)=f_{1}\left(x^{1}\right) \cdots f_{d}\left(x^{d}\right)$, where each $f_{k}$ is a trigonometric polynomial. In fact, we can just take $f_{k}\left(x^{k}\right)=\exp \left(\lambda_{k} x^{k}\right)$. Since the components of $B$ are independent, we just need to prove the formulas only for $d=1$. This can be done easily by induction on $n$, using the Markov property of $B$ and the fact that certain Gaussian integrals can be computed explicitly.

Remark. Observe that

$$
H\left[s_{1}, \ldots, s_{n} ; \gamma_{1}, \ldots, \gamma_{n}\right](t, x, y)=E^{x}\left\{e^{\gamma_{1} \cdot B_{s_{1}}+\cdots+\gamma_{n} \cdot B_{s_{n}}} \mid B_{t}=y\right\} p_{d}(t, x, y)
$$

and, therefore, if we set $y=x+u$, Lemma 2.7 gives

$$
\begin{aligned}
E^{x}\left\{e^{\gamma_{1} \cdot B_{s_{1}}+\cdots+\gamma_{n} \cdot B_{s_{n}}} \mid B_{t}=x+u\right\} p_{d}(t, x, x+u) \\
\left.=e^{\left(\gamma_{1}^{k}+\cdots+\gamma_{n}^{k}\right) \cdot x^{k}} \frac{1}{(2 \pi t)^{d / 2}} \prod_{k=1}^{d} \prod_{j=1}^{n} \exp \left\{-\frac{1}{2 t}\left[\left(\gamma_{1}^{k} s_{1}+\cdots+\gamma_{n}^{k} s_{n}\right)+u^{k}\right)\right]^{2}\right\} \\
\quad \times e^{(1 / 2) s_{j} \gamma_{j}^{k}\left(\gamma_{j}^{k}+2 \gamma_{j+1}^{k}+\cdots+2 \gamma_{n}^{k}\right)} .
\end{aligned}
$$

Theorem 2.8. If $q \in A P\left(R^{d}\right)$, then the kernel $k(t, x, y)$ of the semigroup $e^{-t L}$ is uniformly continuous on $[a, b] \times R^{d} \times R^{d}$, if $0<a<b<\infty$.

Proof. First we consider the case where

$$
q(x)=\sum_{l=1}^{N} a_{l} e^{i \xi_{l} \cdot x}, \quad \xi_{l} \in R^{d}
$$

Formula (1.4) can be written as

$$
k_{n}(t, x, y)=\int_{\mathscr{P}_{t}} E^{x}\left\{q\left(B_{s_{1}}\right) \cdots q\left(B_{s_{n}}\right) \mid B_{t}=y\right\} d s_{1} \cdots d s_{n},
$$

where $\mathscr{P}_{t}$ is the "pyramidoid" $\left\{\left(s_{1}, \ldots, s_{n}\right): 0 \leq s_{1} \leq \cdots \leq s_{n} \leq t\right\}$. The integrand is a finite sum of expressions of the same form as the one in (2.2), with 
all the $\gamma_{j}^{k}$ 's being pure imaginary. Thus, looking at (2.3) (with $u=y-x$ ) we can conclude that $k_{n}(t, x, y)$ is uniformly continuous on $[a, b] \times R^{d} \times R^{d}$. Since the series in (1.3c) converges uniformly, we have finished the trigonometric case. The general case is a consequence of Lemma 2.6 and the first remark following it.

Remark. We do not know whether there is a more direct way to prove Theorem 2.8.

\section{THE KERNELS OF THE POWERS OF THE RESOLVENT}

Let $z$ be a complex number not in $\sigma(L)$. We denote by $G^{m}(x, y ; z)$ the kernel of $(L-z)^{-m}$, where $m$ is a positive integer. In particular, $G^{1}(x, y ; z)=$ $G(x, y ; z)$ is the Green's function that corresponds to $L$. The existence of these kernels and certain of their properties are established in [S.B, Theorem B.7.2]. We summarize few basic estimates in the following theorem.

Theorem 3.1. For any $m=1,2, \ldots, G^{m}(x, y ; z)$ is continuous in $(x, y)$ away from $x=y$. If $|x-y| \geq 1$, then there is $a b>0$ such that

$$
\left|G^{m}(x, y ; z)\right| \leq C(m, z, b) e^{-b|x-y|},
$$

where $C(m, z, b)$ is a constant depending on $m, z$ and $b$. If $\mathfrak{I}\{z\} \geq \delta>0$, then it is a consequence of the resolvent equation that $C$ does not have to depend on $z$. Also, if $l=d-2 m>0$,

$$
\left|G^{m}(x, y ; z)\right| \leq C|x-y|^{-l},
$$

whereas, if $m>d / 2, G^{m}(x, y ; z)$ is jointly continuous in $x$ and $y$ everywhere because of (1.10).

Remark. If $d=1,2$ or 3 , the operator $(L-z)^{-1}-(-\Delta / 2-z)^{-1}$ has a bounded kernel which is jointly continuous and analytic in $z$. For small $\mathfrak{R}\{z\}$, this follows from (1.5), (1.3c) and (1.3a). Then we can use the resolvent equation or basic properties of the Stieltjes transform (see [W]) to extend the result to all $z$ which are not in $\sigma(L) \cup \sigma(-\Delta / 2)$.

Example. To get an idea of how $G(x, y ; z)$ looks, we consider the easiest case, namely $q \equiv 0$. Then $\sigma(L)=[0, \infty)$ and (the index denotes the dimension)

$$
G_{1}(x, y ; z)=\frac{e^{-\sqrt{-2 z}|x-y|}}{\sqrt{-2 z}}, \quad G_{3}(x, y ; z)=\frac{e^{-\sqrt{-2 z}|x-y|}}{2 \pi|x-y|}
$$

and, by elementary properties of Laplace transforms,

$$
G_{d+2}^{m}(x, y ; z)=\frac{1}{2 \pi} \int_{-\infty}^{z} G_{d}^{m}(x, y ; \zeta) d \zeta .
$$

In fact, $G(x, y ; z)$ is an elementary function for any $d$ odd, whereas, if $d$ is even, $G(x, y ; z)$ can be expressed in terms of the modified Bessel functions.

The equation

implies

$$
(L-z)^{-m}=m \int_{-\infty}^{z}(L-\zeta)^{-(m+1)} d \zeta
$$

$$
G^{m}(x, y ; z)=m \int_{-\infty}^{z} G^{m+1}(x, y ; \zeta) d \zeta
$$


(if $x=y$, we must assume that $m>d / 2$ ), from which it follows that $G^{m}(x, y ; z)$ is analytic in $z$, if $z$ is not in the spectrum. If $\mathfrak{R}\{z\}<\lambda_{0}=$ inf $\sigma(L)$, then

$$
(L-z)^{-m}=\int_{0}^{\infty} \cdots \int_{0}^{\infty} e^{\left(t_{1}+\cdots+t_{m}\right) z} k\left(t_{1}+\cdots+t_{m}, x, y\right) d t_{1} \cdots d t_{m}
$$

and so

$$
G^{m}(x, y ; z)=\int_{0}^{\infty} \cdots \int_{0}^{\infty} e^{\left(t_{1}+\cdots+t_{m}\right) z} k\left(t_{1}+\cdots+t_{m}, x, y\right) d t_{1} \cdots d t_{m} .
$$

Furthermore, formulas $(1.11),(1.12)$ and analytic continuation imply that, for all $z$ not in $\sigma(L)$ and $m>d / 2$,

$$
G^{m}(x, x ; z)=\int_{\lambda_{0}}^{\infty} \frac{d_{\lambda} e(x, x ; \lambda)}{(\lambda-z)^{m}} .
$$

From now on we assume $q \in A P\left(R^{d}\right)$ and $m>d / 2$. Our goal is to show that, in this case, $G^{m}(x, x ; z)$ is almost periodic in $x$ with frequency module not exceeding $F M(q)$. But, first, we need a few lemmas.

Lemma 3.2. Let $f(s, x)$ be (jointly) uniformly continuous on $[a, b] \times R^{d}$ and almost periodic in $x$, with frequencies in $F M(q)$, for all $s \in[a, b]$. Then

$$
\varphi(x)=\int_{a}^{b} f(s, x) d s
$$

is almost periodic and $F M(\varphi) \subset F M(q)$.

Proof. Let $N$ be a positive integer. We divide $[a, b]$ into $N$ equal subintervals separated by the points

$$
s_{j}=a+\frac{(b-a) j}{N}, \quad j=0,1, \ldots, N .
$$

Now we approximate $f(s, x)$ by the "s-polygonal" function $f_{N}(s, x)$ defined by

$$
f_{N}(s, x)=f\left(s_{j}, x\right)+\frac{N}{b-a}\left[f\left(s_{j+1}, x\right)-f\left(s_{j}, x\right)\right]\left(s-s_{j}\right) .
$$

Notice that $f_{N}(s, x)$ is almost periodic in $x$, with frequency module included in $F M(q)$, and that

$$
\lim _{N} f_{N}(s, x)=f(s, x), \quad \text { uniformly in }(s, x) .
$$

Next we set

$$
\begin{aligned}
\varphi_{N}(x) & =\int_{a}^{b} f_{N}(s, x) d s \\
& =\left[f\left(s_{0}, x\right)+2 f\left(s_{1}, x\right)+\cdots+2 f\left(s_{N-1}, x\right)+f\left(s_{N}, x\right)\right] \frac{b-a}{2 N}
\end{aligned}
$$

and observe that $\varphi_{N}$ is in $A P\left(R^{d}\right)$, with $F M\left(\varphi_{N}\right) \subset F M(q)$. Finally

$$
\left|\varphi(x)-\varphi_{N}(x)\right| \leq \int_{a}^{b}\left|f(s, x)-f_{N}(s, x)\right| d s
$$


and therefore, by (3.3),

$$
\lim _{N} \varphi_{N}(x)=\varphi(x), \quad \text { uniformly in } x .
$$

Lemma 3.3. We set $D=\{z \in C:|z|<1\}$. Let $g(x, z)$ be bounded and continuous on $R^{d} \times D$ and, for each $x \in R^{d}$, analytic in $D$, as a function of $z$. We, also, assume that there is a simple closed and piecewise differentiable curve $\Gamma$ in $D$ such that $g(x, z)$ is jointly uniformly continuous on $R^{d} \times \Gamma$ and almost periodic in $x$, with frequency module included in $F M(q)$. Then, for all $z \in D, g(x, z)$ is almost periodic in $x$, with frequencies in $F M(q)$.

Proof. Since $g(x, z)$ is $z$-analytic in $D$ we must have

$$
g(x, z)=\sum_{n=0}^{\infty} a_{n}(x) z^{n}, \quad z \in D,
$$

and so, if $0<r<1$, our assumptions together with Parseval's formula give

$$
\sum_{n=0}^{\infty}\left|a_{n}(x)\right|^{2} r^{2 n}=\frac{1}{2 \pi} \int_{0}^{2 \pi}\left|g\left(x, \mathrm{re}^{i \theta}\right)\right|^{2} d \theta \leq M^{2},
$$

where $M$ is a bound for $|g(x, z)|$ on $R^{d} \times D$. Letting $r \uparrow 1$, we obtain

$$
\sum_{n=0}^{\infty}\left|a_{n}(x)\right|^{2} \leq M^{2}
$$

In particular,

$$
\left|a_{n}(x)\right| \leq M, \text { for all } n \text {. }
$$

Now, let $G$ be the interior of $\Gamma$. Without loss of generality (by using a conformal automorphism of $D$ ) we can assume $0 \in G$. Then, by the Cauchy integral formulas,

$$
a_{n}(x)=\frac{1}{2 \pi i} \int_{\Gamma} \frac{g(x, z)}{z^{n+1}} d z
$$

and therefore, by Lemma 3.2, $a_{n}(x)$ is almost periodic in $x$, with $F M\left(a_{n}\right) \subset$ $F M(q)$. Finally, for each $z \in D,(3.5)$ implies that the series in (3.4) converges uniformly in $x$ and so, we are done.

Remark. Because of the Riemann mapping theorem, in Lemma 3.3 we could take $D$ to be any simply connected open subset of the complex numbers.

Now, we are ready to prove our theorem.

Theorem 3.4. Let $q \in A P\left(R^{d}\right)$ and $m>d / 2$. Then, for $z \in C \backslash \sigma(L)$, $G^{m}(x, x ; z)$ is almost periodic in $x$, with frequency module not exceeding $F M(q)$.

Proof. If $\mathfrak{R}\{z\}<\lambda_{0}$ the result follows by applying Lemma 3.2 to (3.1). In fact, by $(3.1)$,

$$
G^{m}(x, x ; z)=\lim _{\substack{M \uparrow \infty \\ \varepsilon \downarrow 0}} \int_{\varepsilon}^{M} \cdots \int_{\varepsilon}^{M} e^{\left(t_{1}+\cdots+t_{m}\right) z} k\left(t_{1}+\cdots+t_{m}, x, x\right) d t_{1} \cdots d t_{m} .
$$

The quantity inside the limit is almost periodic in $x$ by Theorems $2.5,2.8$ and Lemma 3.2 and the above limit is uniform, for $\mathfrak{R}\{z\} \leq \lambda<\lambda_{0}$ by (1.7) and 
the fact that (see [S.B]), for any $\mu<\lambda_{0}, k(t, x, y)=o\left(e^{-\mu t}\right)$ as $t \rightarrow \infty$, uniformly in $(x, y)$.

The rest of the theorem is then a corollary of Lemma 3.3. Because, by (3.1) $G^{m}(x, x ; z)$ is jointly uniformly continuous if $\Re\{z\} \leq \lambda<\lambda_{0}$ and, if we take $D$ to be any simply connected domain such that $\bar{D}$ is compact and lies inside the open upper (or lower) half plane, then $G^{m}(x, x ; z)$ is (jointly continuous and) bounded and $z$-analytic in $D$ by (3.2).

Remarks. (a) If $u \in R^{d}$ and $u \neq 0$, then a similar argument shows that $G^{m}(x, x+u ; z)$ is almost periodic in $x$ with frequencies in $F M(q)$, for any $m=1,2, \ldots$, as long as $z$ is not in the spectrum of $L$. If $m \leq d / 2$, we can not derive the boundedness of $G^{m}(x, x+u ; z)$ on $R^{d} \times D$ from (3.2), but we can use Theorem 3.1 instead.

(b) For $d=1,2$ or 3 , using similar arguments we can show that the kernel $G(x, y ; z)-G_{d}(x, y ; z)$ of $(L-z)^{-1}-(-\Delta / 2-z)^{-1}$ (recall the remark following Theorem 3.1) is almost periodic in $x$, if $y=x$.

\section{THE COMPLEX ROTATION NUMBER AND THE INTEGRATED DENSITY OF STATES}

We start with an equation we derived in $\S 1$, namely

$$
k(t, x, x)=\int_{\lambda_{0}}^{\infty} e^{-\lambda t} d_{\lambda} e(x, x ; \lambda)
$$

Now, let $C(T)$ be a cube in $R^{d}$ with side $T$ center at the origin and sides parallel to the axes (as in Theorem 2.2). We set

$$
k(t, T)=\frac{1}{T^{d}} \int_{C(T)} k(t, x, x) d x \quad \text { and } \quad e(T, \lambda)=\frac{1}{T^{d}} \int_{C(T)} e(x, x ; \lambda) d x .
$$

Notice that $e(T, \lambda)$ is nondecreasing in $\lambda$. If $T_{n} \uparrow \infty$, then $k\left(t, T_{n}\right) \rightarrow$ $M_{x}[k(t, x, x)]$ because of Theorem 2.5. By Helly's extraction theorem, there is a subsequence of $T_{n}$ for which $e\left(T_{n} ; \lambda\right)$ converges to some limit, say $\alpha(\lambda)$. Then, by (1.11) we must have

$$
M_{x}[k(t, x, x)]=\int_{\lambda_{0}}^{\infty} e^{-\lambda t} d \alpha(\lambda),
$$

hence, by the uniqueness of Laplace transforms we have proved the following.

Theorem 4.1. The mean value of $e(x, x ; \lambda)$

$$
\alpha(\lambda)=M_{x}[e(x, x ; \lambda)]
$$

exists.

Notice that $\alpha(\lambda)$ increases only if $\lambda \in \sigma(L)$, being constant outside the spectrum and, by (1.12),

$$
\alpha(\lambda) \leq C_{1} \lambda_{+}^{d / 2}+C_{2},
$$

where $\lambda_{+}=\max \{\lambda, 0\}$. If $d=1$, then $\pi \alpha(\lambda)$ is the rotation number of [J-M], where it is shown that it is continuous in $\lambda$, it is an invariant quantity for all solutions of $(L-\lambda) u=0$ (it is the average number of rotations of the point $\left(u(x), u^{\prime}(x)\right)$, as $x$ runs through the real axis and this justifies its name) and, 
if $\lambda$ is not in $\sigma(L)$, then $2 \pi \alpha(\lambda) \in F M(q)$. Are there corresponding properties in the multidimensional case?

Now, we consider the operator $L^{T}=L$ acting on $L^{2}[C(T)]$ with zero boundary conditions and let $k^{T}(t, x, y)$ and $e^{T}(x, y ; \lambda)$ be the kernels of $e^{-t L^{T}}$ and $E_{\lambda}^{T}$ respectively. In $\S 1$, formula $(1.13)$, we say that

$$
k^{T}(t, x, y) \uparrow k(t, x, y), \quad \text { as } t \rightarrow \infty,
$$

which implies

$$
k^{T}(t, T) \leq k(t, T),
$$

where we have used the notation of (4.1). On the other hand, if $\sigma_{T}$ is the exit time of $C(T)$, then, given $\varepsilon>0$, we can choose $T$ large enough to assure that

$$
P^{x}\left\{\sigma_{T}<t \mid B_{t}=x\right\}<\varepsilon, \quad \text { if } x \in C(T-\sqrt{T}),
$$

which implies that, if $t>0$ is fixed and if $x \in C(T-\sqrt{T}$ ), then (by using the probabilistic expressions of the kernels)

$$
\begin{aligned}
k(t, x, x)-k^{T}(t, x, x) & =E^{x}\left\{e_{q}(t) 1_{\left[\sigma_{T} \leq t\right]} \mid B_{t}=x\right\} p_{d}(t, x, x) \\
& \leq e^{\|q\|^{t}} \varepsilon \frac{1}{(2 \pi t)^{d / 2}}=Q_{t} \varepsilon .
\end{aligned}
$$

Then

$$
\begin{aligned}
k^{T}(t, T) & \geq k^{T}(t, T-\sqrt{T}) \geq k(t, T-\sqrt{T})-Q_{t} \varepsilon \\
& \geq k(t, T)-O\left(T^{-1 / 2}\right)-Q_{t} \varepsilon, \quad \text { as } T \rightarrow \infty .
\end{aligned}
$$

Since $\varepsilon$ is arbitrary, the above inequality together with (4.5) imply the following result.

Theorem 4.2. For each $t>0$,

$$
\lim _{T \uparrow \infty} \frac{1}{T^{d}} \int_{C(T)} k^{T}(t, x, x) d x=M_{x}[k(t, x, x)]
$$

and therefore

$$
\lim _{T \uparrow \infty} \frac{1}{T^{d}} \int_{C(T)} e^{T}(x, x ; \lambda) d x=\alpha(\lambda) .
$$

Equation (4.7) follows from (4.6) by our standard selection argument and implies that $\alpha(\lambda)$ is the integrated density of states of [A-S].

The following definition is legitimate because of Theorem 3.4.

Definition. For $m>d / 2$ and $z$ not in the spectrum of $L$ we define the $m$ th order complex rotation number to be

$$
w_{m}(z)=M_{x}\left[G^{m}(x, x ; z)\right] .
$$

Using (3.2), (1.12) and Theorem 4.1 in (4.8) we obtain

$$
w_{m}(z)=\int_{\lambda_{0}}^{\infty} \frac{d \alpha(\lambda)}{(\lambda-z)^{m}},
$$


hence, by $(4.2), w_{m}(z)$ is analytic outside $\sigma(L)$. In fact, we can differentiate (4.9) and get

$$
w_{m}^{\prime}(z)=m w_{m+1}(z) .
$$

The above imply that we can define $w_{m}(z)$ modulo a polynomial, even if $m \leq$ $d / 2$ because, for $1 \leq m \leq d / 2$, we can just set (in the spirit of (4.10))

$$
w_{m}(z)=m \int w_{m+1}(z) d z
$$

and, for $m=0$, we can simply define

$$
w(z)=w_{0}(z)=\int w_{1}(z) d z .
$$

We could call $w(z)$ the complex rotation number. In the one-dimensional case, this is the same $w(z)$ that appears in [J-M], where it is shown that it has some interesting properties, namely

$$
\lim _{\mu \downarrow 0} \mathfrak{I}\{w(\lambda+i \mu)\}=\pi \alpha(\lambda)
$$

and

$$
\frac{\delta w(z ; q)}{\delta q}=G(x, x ; z)
$$

where in the left-hand side we have a functional derivative of $w$. The variation of $q$ is restricted in $A P\left(R^{1}\right)$ and to have frequencies in $F M(q)$.

If $d<4$ we can get something very close to (4.13) by Remark (b) following Theorem 3.4, a basic estimate for Stieltjes transforms and the inversion formula for these transforms. For $d \geq 4$, we only have (4.9), but we expect that (4.13) remains true up to a polynomial term.

The extension of (4.14) to higher dimensions $(d=1$ is included) is given by the following theorem.

Theorem 4.3. If $m+1>d / 2$, then

$$
\frac{\delta w_{m}(z ; q)}{\delta q}=G^{m+1}(x, x ; z)
$$

where the precise meaning of (4.15) is that

$$
\left.\frac{d}{d \varepsilon} w_{m}(z ; q+\varepsilon p)\right|_{\varepsilon=0}=M_{x}\left[G^{m+1}(x, x ; z) p(x)\right]
$$

is true for all $p$ in $A P\left(R^{d}\right)$.

We need first the following lemma which reminds one of the Tonelli-Fubini theorem.

Lemma 4.4. Let $p \in A P\left(R^{d}\right)$ and $0<s<t$. Then

$$
M_{x}\left[\int_{R^{d}} k(t-s, x, z) p(z) k(s, z, x) d z\right]=M_{z}[k(t, z, z) p(z)] .
$$


Proof. Let $C(T)$ be as usual. By (1.7), $k(t, x, z)$ is very small if $x$ and $z$ are very far apart and hence we have

$$
\begin{aligned}
\lim _{T \uparrow \infty} & \frac{1}{T^{d}} \int_{C(T)} \int_{R^{d}} k(t-s, x, z) p(z) k(s, z, x) d z d x \\
& =\lim _{T \uparrow \infty} \frac{1}{T^{d}} \int_{C(T-\sqrt{T})} \int_{R^{d}} k(t-s, x, z) p(z) k(s, z, x) d z d x \\
& =\lim _{T \uparrow \infty} \frac{1}{T^{d}} \int_{C(T-\sqrt{T})} \int_{C(T)} k(t-s, x, z) p(z) k(s, z, x) d z d x \\
& =\lim _{T \uparrow \infty} \frac{1}{T^{d}} \int_{C(T)} \int_{C(T)} k(t-s, x, z) p(z) k(s, z, x) d z d x \\
& =\lim _{T \uparrow \infty} \frac{1}{T^{d}} \int_{C(T)} p(z)\left[\int_{C(T)} k(t-s, x, z) k(s, z, x) d x\right] d z \\
& =\lim _{T \uparrow \infty} \frac{1}{T^{d}} \int_{C(T-\sqrt{T})} p(z)\left[\int_{C(T)} k(t-s, x, z) k(s, z, x) d x\right] d z \\
& =\lim _{T \uparrow \infty} \frac{1}{T^{d}} \int_{C(T-\sqrt{T})} p(z)\left[\int_{R^{d}} k(t-s, x, z) k(s, z, x) d x\right] d z .
\end{aligned}
$$

The rest follows from (1.8), i.e. the Chapman-Kolmogorov equation.

Proof of Theorem 4.3. We define

Thus, by (1.6),

$$
\kappa(t ; q)=\boldsymbol{H}_{x}[k(t, x, x ; q)] \text {. }
$$

$$
\begin{aligned}
\kappa(t ; q+\varepsilon p) & =M_{x}[k(t, x, x ; q+\varepsilon p)] \\
& =M_{x}\left[E^{x}\left\{e_{q}(t) e_{\varepsilon p}(t) \mid B_{t}=x\right\}(2 \pi t)^{-d / 2}\right] \\
& =M_{x}\left[E^{x}\left\{e_{q}(t)\left(1+\varepsilon \int_{0}^{1} p\left(B_{s}\right) d s\right) \mid B_{t}=x\right\}(2 \pi t)^{-d / 2}\right]+O\left(\varepsilon^{2}\right) .
\end{aligned}
$$

Therefore

$$
\left.\frac{d}{d \varepsilon} \kappa(t ; q+\varepsilon p)\right|_{\varepsilon=0}=M_{x}\left[\int_{0}^{t} E^{x}\left\{e_{q}(t) p\left(B_{s}\right) \mid B_{t}=x\right\}(2 \pi t)^{-d / 2} d s\right] .
$$

Using the Markov property of $B$, the above equation becomes

$$
\left.\frac{d}{d \varepsilon} \kappa(t ; q+\varepsilon p)\right|_{\varepsilon=0}=M_{x}\left[\int_{0}^{t} \int_{R^{d}} k(t-s, x, z) p(z) k(s, z, x) d z d s\right] .
$$

If instead of $\int_{0}^{t}(\cdots) d s$ above, we write $\int_{\delta}^{t-\delta}(\cdots) d s$, where $\delta>0$ is small, everything changes by $O(\delta)$ and we can move $M_{x}$ inside the new integral by bounded convergence (and almost periodicity). Hence (since $\delta$ is arbitrary) the above equation becomes

$$
\left.\frac{d}{d \varepsilon} \kappa(t ; q+\varepsilon p)\right|_{\ell=0}=\int_{0}^{t} M_{x}\left[\int_{R^{d}} k(t-s, x, z) p(z) k(s, z, x) d z\right] d s
$$

and, with the help of (4.16), we can conclude that

$$
\left.\frac{d}{d \varepsilon} \kappa(t ; q+\varepsilon p)\right|_{e=0}=M_{x}[t k(t, x, x) p(x)], \quad \text { i.e. } \frac{\delta \kappa(t ; q)}{\delta q}=t k(t, x, x) .
$$


Therefore, if $\mathfrak{R}\{z\}<\lambda_{0}$, formula (3.1) finishes the proof, since the differentiation with respect to $\varepsilon$ inside the multiple integral appearing in (3.1) is easily justified. For the other $z$ 's the theorem can be proved by an analytic continuation argument that resembles the one used for Lemma 3.3.

Examples. 1. If $q \equiv 0$, then

$$
\alpha(\lambda)=\frac{\lambda^{d / 2}}{(2 \pi)^{d / 2} \Gamma(d / 2+1)}, \quad \text { if } \lambda \geq 0,
$$

(of course $\alpha(\lambda)=0$, if $\lambda<0$ ) and

$$
\begin{gathered}
w(z)=-\frac{z^{d / 2} \ln (-z)}{(2 \pi)^{d / 2}(d / 2) !}, \quad \text { if } d \text { is even, } \\
w(z)=\frac{(-z)^{d / 2} \sqrt{2}}{\pi^{(d-1) / 2} 1 \cdot 3 \cdot 5 \cdots d}, \quad \text { if } d \text { is odd },
\end{gathered}
$$

modulo a polynomial of degree at most $d / 2$. Thus, (4.13) is satisfied in the case $q \equiv 0$, assuming $\ln (-1)=-\pi i$.

2. Let $q_{1}, q_{2} \in A P\left(R^{1}\right)$ having rotation numbers $\alpha_{1}(\lambda)$ and $\alpha_{2}(\lambda)$ respectively. Without loss of generality, we can assume that the corresponding spectra are nonnegative. Now, we form the two-dimensional potential $q(x, y)=q_{1}(x)+q_{2}(y)$. This is a separable case and the corresponding to $\alpha$ is given by the convolution

$$
\alpha(\lambda)=\int_{0}^{\lambda} \alpha_{1}(\lambda-\mu) d \alpha_{2}(\mu)
$$

\section{APPENDIX}

Here we give the proof of (1.11). Without loss of generality we assume that $\lambda_{0}=0$.

Theorem. Let the notation be as in $\S 1$. Then

$$
k(t, x, y)=t \int_{0}^{\infty} e^{-\lambda t} e(x, y ; \lambda) d \lambda .
$$

(This implies (1.11) immediately, since $e(x, x ; \lambda)$ is nondecreasing in $\lambda$.

Proof. Let $\tau=t+i s$, with $t>0$. Then, $e^{-\tau \lambda}$ satisfies (1.10), so the operator $e^{-\tau L}$ has a kernel, which we denote by $k(\tau, x, y)$. This kernel is jointly continuous in $x, y$ and $\tau$ and analytic in $\tau$, because $f(\lambda)=-\lambda e^{-\tau \lambda}=(d / d \tau) e^{-\tau \lambda}$ satisfies (1.10) too. Furthermore, if $t \geq t_{0}>0, k(\tau, x, y)$ is bounded uniformly in $(\tau, x, y)$, since

$$
\begin{aligned}
|k(\tau, x, y)| & \leq\left\|e^{-\tau L}\right\|_{1, \infty} \leq\left\|e^{-(t / 2) L}\right\|_{2, \infty}\left\|e^{i s L}\right\|_{2,2}\left\|e^{-(t / 2) L}\right\|_{1,2} \\
& =\left\|e^{-(t / 2) L}\right\|_{2, \infty}\left\|e^{-(t / 2) L}\right\|_{1,2} .
\end{aligned}
$$

Therefore, the kernel

$$
a(x, y ; \lambda)=\frac{e^{\lambda t_{1}}}{2 \pi} \int_{-\infty}^{\infty} \frac{k\left(t_{1}+i s, x, y\right)}{\left(t_{1}+i s\right)^{2}} e^{i \lambda s} d s, \quad\left(t_{1}>0 \text { is fixed }\right)
$$

is jointly continuous in $x, y$ and $\lambda$, uniformly bounded, if $\lambda$ is bounded above and $\sup _{x, y}|a(x, y ; \lambda)|=O\left(e^{\lambda t_{1}}\right)$, as $\lambda \rightarrow \infty$ (observe that $t_{1}>0$ is arbitrary). 
For each $\lambda$, the integral operator with kernel $a(x, y ; \lambda)$ is in $\mathscr{L}\left(L^{p}, C_{b}\right)$, $1 \leq p \leq \infty$, because $e^{-\tau L}$ belongs to all these classes. We also have (by Integral Transform Theory-see for example [W]) that, for all $t>t_{1}$ and therefore for all $t>0$, since $t_{1}>0$ is arbitrary,

$$
\frac{k(t, x, y)}{t^{2}}=\int_{-\infty}^{\infty} e^{-\lambda t} a(x, y ; \lambda) d \lambda .
$$

Next we notice that the operator

$$
A_{\lambda}=\int_{-\infty}^{\lambda} E_{\mu} d \mu=\int_{0}^{\lambda} E_{\mu} d \mu
$$

has a kernel by (1.10) and satisfies

$$
\frac{e^{-t L}}{t^{2}}=\int_{0}^{\infty} e^{-\lambda t} A_{\lambda} d \lambda=\int_{-\infty}^{\infty} e^{-\lambda t} A_{\lambda} d \lambda
$$

Comparing this with (A.2), we get (by the properties of $a(x, y ; \lambda)$ and $A_{\lambda}$ mentioned above and the uniqueness for bilateral Laplace transforms-see [W]) that $a(x, y ; \lambda)$ is the kemel of $A_{\lambda}$. Thus

$$
a(x, y ; \lambda)=\int_{0}^{\lambda} e(x, y ; \mu) d \mu,
$$

which, together with (A.2) implies (A.1).

Remark. We can construct $k(\tau, x, y)$ using formulas similar to (1.3) and procedures analog to the ones of Theorem 2.8. This construction implies that, if $q \in A P\left(R^{d}\right), k(\tau, x, x+u)$ is almost periodic in $x$, for all $u \in R^{d}$, with frequencies in $F M(q)$, and uniformly continuous on $K \times R^{d} \times R^{d}$, where $K$ is a compact subset of the right half plane. Then, using the arguments of $\S 3$, we can obtain that $a(x, x+u ; \lambda)$, as defined in the above proof, is almost periodic in $x$ too.

\section{ACKNOWLEDGMENTS}

We want to thank Professor George Papanicolaou for his encouragement and suggestions. Also Professors Barry Simon, Russell Johnson, Persi Deift and Stefanos Venakides for helpful discussions (the discussions with B. Simon and $\mathrm{R}$. Johnson were held by telephone).

\section{REFERENCES}

[A-S] J. Avron and B. Simon, Almost periodic Schrödinger operators. II. The integrated density of states, Duke Math. J. 50 (1983), 369-391.

[C-L] E. Coddington and N. Levinson, Theory of ordinary differential equations, McGraw-Hill, New York, 1955.

[F] W. Feller, An introduction to probability theory and its applications, vol. II, 2nd ed., Wiley, New York, 1971.

[J-M] R. Johnson and J. Moser, The rotation number for almost periodic potentials, Comm. Math. Phys. 84 (1982), 403-438; erratum: Comm. Math. Phys. 90 (1983), 317-318.

[L] L. Loomis, Abstract harmonic analysis, Van Nostrand, Princeton, N.J., 1953.

[S.B] B. Simon, Schrödinger semigroups, Bull. Amer. Math. Soc. (N.S.) 7 (1982), 447-526. 
[S.G] G. Scharf, Fastperiodische Potentiale, Helv. Phys. Acta 24 (1965), 573-605.

[S.M] M. A. Shubin, Almost periodic functions and partial differential operators, Russian Math. Surveys 33:2 (1978), 1-52.

[W] D. V. Widder, The Laplace transform, Princeton Univ. Press, Princeton, N.J., 1941.

Department of Mathematics, Duke University, Durham, North Carolina 27706 